\title{
Research on the Method to Calibrate Structure Parameters of Line Structured Light Vision Sensor
}

\author{
Mingang Niu, a , Kangnian Zhao, b, Jie Wan ${ }^{2, c}$, Yanyan $\mathrm{Li}^{3, \mathrm{~d}}$, Shuzhong $\mathrm{Liu}^{4, \mathrm{e}}$ \\ ${ }^{1}$ Department of Geological Engineering of Qinghai University, Xining, 810000, China \\ ${ }^{2}$ School of Earth \& Space Sciences, Peking University, Beijing, 100000, China \\ ${ }^{3}$ School of Software \& Microelectronics, Peking University, Beijing, 100000, China \\ ${ }^{4}$ School of Resource environment \& planning of Dezhou University, Dezhou, 253000, China \\ a18797396784@163.com, b13519755989@139.com, cwhuwan@163.com, \\ diyan.@pku.edu.cn, ${ }^{\mathrm{e}} 13589943826 @ 163 . c o m$
}

Keywords: Linear structured light, Camera, Retro-reflective target, Light plane

\begin{abstract}
The existing linear structured light sensor system calibration process to the equipment requirement is high, the calibration process such complicated problems, this paper puts forward a simple calibration method. Use of the most commonly used transit system, back light reflection artificial materials, as well as the independent development of photography calibration software, in a plane wall master three fine line, at the same time in space layout a filament, and the four o 'clock in the transit system of coordinates. Combined with camera coordinate system in the transit system of bearing, the light plane and unified camera, so as to get the light plane point in the camera coordinate system coordinates. Structured light system can be moved several position, get more sets of light plane, thus to find the mean value. The experimental data show that field calibration.
\end{abstract}

\section{Introduction}

Line structured light active sensing technology with low cost, volume small, non-contact, fast speed, convenient and flexible and light stripe image information easy extraction, etc. advantage, have important significance and broad application prospects in visual measurement, industrial inspection [1]. The basic principle is that the structure light stripe is modulated by the height of the measured object, camera by acquiring the deformation stripe image information, according to the relative position relationship between the camera and the laser to solve the $3 \mathrm{D}$ information of the object surface [2]. Therefore, the calibration of line structured light sensing system is one of the key links in the whole test task.

At present, a lot of calibration methods are proposed according to the measurement model of the line structured light sensing system. The main methods have drawing calibration method [3], the calibration method of based on three-dimensional targets and cross ratio invariability $[4,5]$ and tooth shape target method $[6,7]$, these three kinds of calibration methods all need the precision calibration auxiliary equipment, the calibration process is tedious, and is not suitable for the spot calibration.

In order to quickly and easily find out the structure light sensor model, this paper presents a calibration method based on the combination of the theodolite and the existing software. This method is simple in operation and can be implemented in a simple environment.

\section{Structural Light Measurement Principle}

Line structured light detection system consists of laser, camera and the point of view of the scanning equipment general, laser projects a light plane to the measured object surface, modulated by depth variation of the measured object surface, a deformation of the structured light stripe is formed, by the camera gets stripe of 2D image.

The relationship between image points of camera image plane and object points of the laser light plane to meet the camera coordinate system of the collinear equation: 


$$
\left.\begin{array}{l}
x=-f \frac{a_{1}\left(X-X_{S}\right)+b_{1}\left(Y-Y_{S}\right)+c_{1}\left(Z-Z_{S}\right)}{a_{3}\left(X-X_{S}\right)+b_{3}\left(Y-Y_{S}\right)+c_{3}\left(Z-Z_{S}\right)} \\
y=-f \frac{a_{2}\left(X-X_{S}\right)+b_{2}\left(Y-Y_{S}\right)+c_{2}\left(Z-Z_{S}\right)}{a_{3}\left(X-X_{S}\right)+b_{3}\left(Y-Y_{S}\right)+c_{3}\left(Z-Z_{S}\right)}
\end{array}\right\}
$$

The equation of the light plane in the camera coordinate system is:

$$
a \cdot X+b \cdot Y+c \cdot Z+d=0
$$

If the intrinsic parameters of the camera and the light plane equation are known, according to the formula (1) - (2), we can only determine the coordinates of the $\mathrm{P}$ of the object point.

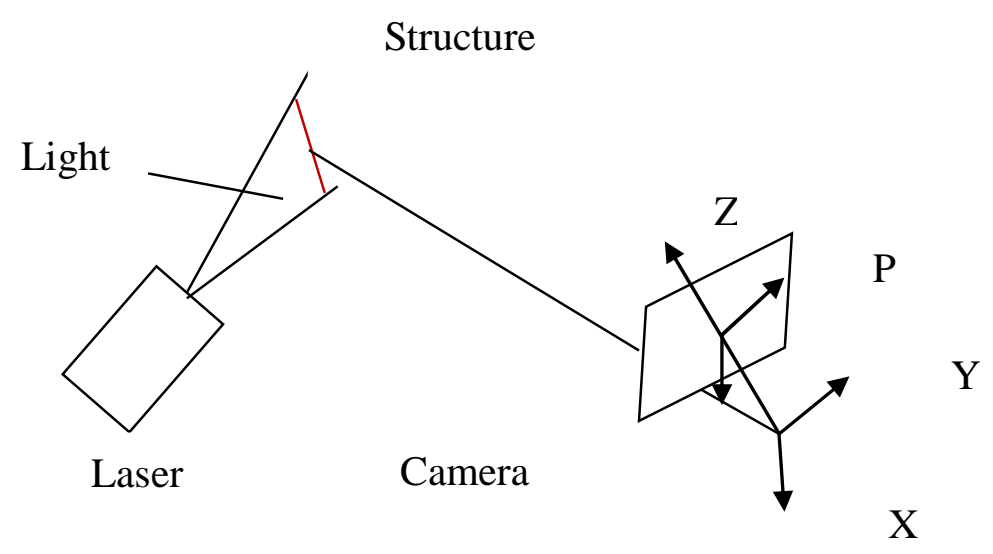

Fig. 1 Design of the Chip Kick Mechanics.

\section{Line Structured Light Sensor Calibration}

The calibration of line structured light sensor system consists of two parts: the calibration of camera's internal parameters and the calibration of the light plane. This section focuses on the calibration method of the light plane.

Retro reflective targets used to implement the measurement of high precision industrial photography and special photography, attached to the measured object on the surface of a kind of artificial marks.

Under the irradiation of a particular position light source (light source can be a flash or a given direction of the infrared light, etc.). It in reflection brightness is white diffuse sign hundreds of thousands of times for features. Binarization image can be got. At the same time, the photographic measurement software, to obtain the photo processing, through the edge extraction, central processing, and so on, through the bundle adjustment get the object coordinates of the mark point.

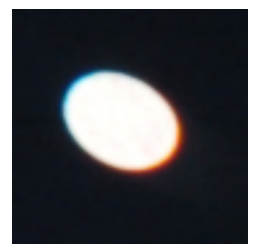

Fig. 2 General mark.

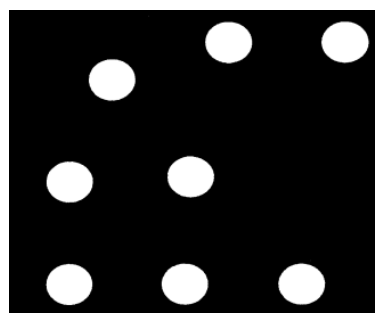

Fig. 3 Point encoding.

The measuring system is composed of two electronic theodolites, which is composed of intelligent industrial measuring system software, by means of mutual aim, a unified measurement coordinate system is established. Unified into the first theodolite coordinate system. 


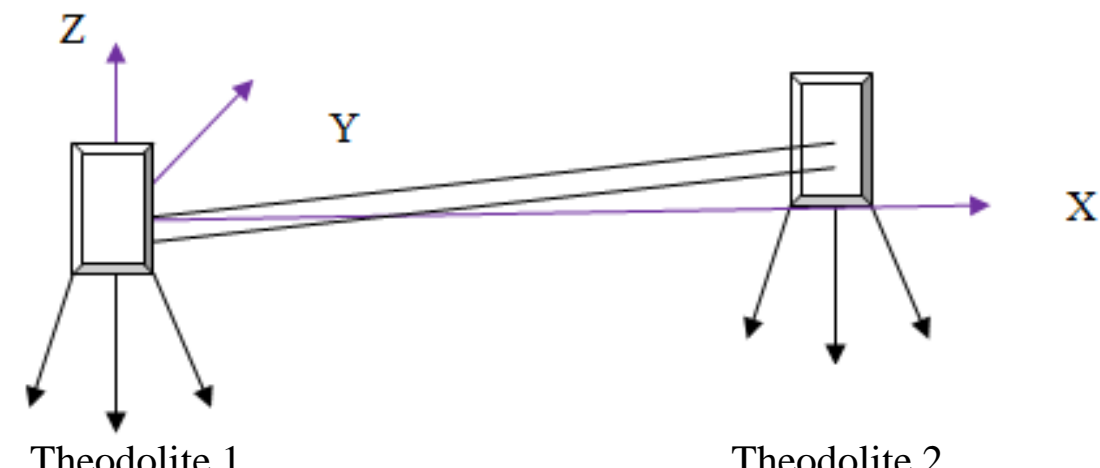

Theodolite 1

Fig. 4 Theodolite coordinate system.

Retro reflective marking materials be pasted on the smooth surface of the wall, choose one of the four major types of common markers as theodolite to aim at the common point. Draw three lines on the wall, and pull a wire between the wall and the wire.

Taking a picture of the reflective logo on the wall with a Nikon camera, get a number of photos, then use photogrammetry software handle these picture, get the 3D coordinates of all points in the camera coordinate system, as the next step data file to be applied to. Then, connecting with two sets of theodolite, we can construct unified theodolite measuring coordinate system by industrial measurement software, by aiming at the center of the four marks on the wall, get their coordinates in the theodolite. These four points can play the role of common point, the conversion of all points on the wall can be converted to the coordinates of the theodolite by common point transformation. At the same time, on the wall camera take pictures on the mark points, the relationship between camera coordinate system and the theodolite coordinate system can be obtained, namely, the transformation parameters of two coordinate systems are obtained, $\mathrm{x}, \mathrm{y}, \mathrm{z}, \varpi, \psi, \kappa$, laser outgoing light plane and four filaments will have four coplanar points in space. At the same time, the position information of the laser surface in the theodolite can be obtained by pointing at four points. By means of software matching and processing, we can get the light plane equation of the light plane in the camera coordinate system.

Several groups of equations can be obtained by turn the orientation of structured light system.

Experimental scene layout as shown in the figure:

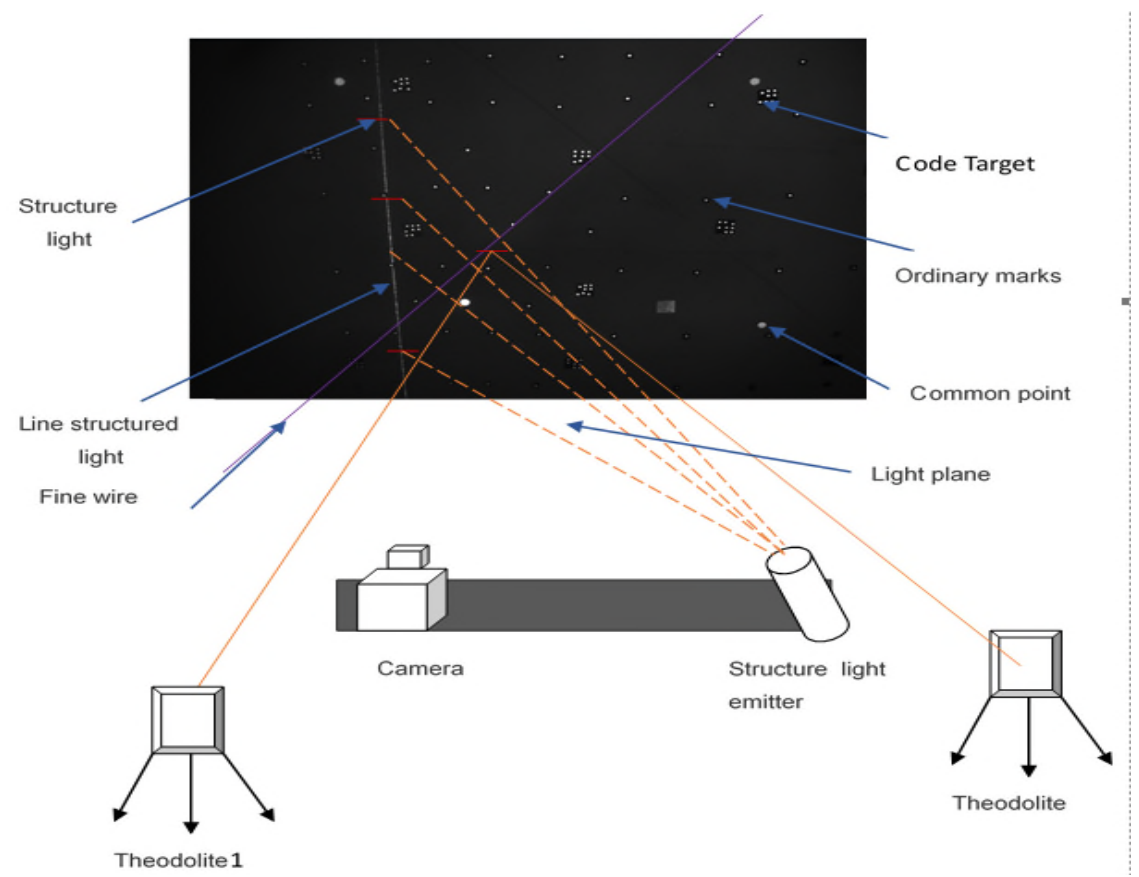

Fig. 5 Experimental layout. 


\section{Experimental Data Processing and Accuracy Analysis}

Using the photos taken by Nikon to deal with, and after public point conversion, get the calibration field data, used to determine the location of the camera information, the data are as follows:

Table 1. Calibration field data.

\begin{tabular}{cccc}
\hline CODE74 & 1759.825 & -2628.009 & 395.708 \\
\hline CODE75 & 1770.274 & -2632.173 & -354.281 \\
CODE78 & 1017.473 & -2579.686 & -668.903 \\
CODE80 & 1013.777 & -2577.028 & 425.323 \\
CODE81 & 1015.711 & -2578.942 & -392.524 \\
CODE84 & 1513.130 & -2612.938 & 129.955 \\
CODE86 & 363.486 & -2531.025 & 443.290 \\
CODE87 & 296.960 & -2527.079 & -409.308 \\
CODE90 & 635.209 & -2550.831 & 197.026 \\
CODE93 & 985.235 & -2577.569 & -1008.338 \\
CODE94 & 1405.377 & -2606.027 & -137.019 \\
CODE95 & 562.560 & -2545.981 & -121.349 \\
CODE96 & 663.989 & -2553.290 & -361.015 \\
NUGGET 46_4 & 1487.274 & -2613.246 & -581.990 \\
NUGGET 46_6 & 1472.069 & -2612.201 & -580.773 \\
NUGGET 46_10 & 1460.026 & -2611.360 & -609.949 \\
NUGGET 46_A & 1457.158 & -2611.144 & -579.535 \\
NUGGET 46_B & 1474.764 & -2612.369 & -616.648 \\
NUGGET 46_C & 1490.029 & -2613.430 & -617.614 \\
NUGGET 46_D & 1491.098 & -2613.491 & -602.998 \\
P1 & 1538.070 & -2614.068 & 248.009 \\
P2 & 1066.881 & -2581.146 & 169.676 \\
P3 & 698.414 & -2555.019 & 165.193 \\
P4 & 390.232 & -2533.727 & -655.701 \\
P5 & 997.797 & -2577.824 & -742.570 \\
P6 & 1438443 & -2609.856 & -647.622 \\
TARGET1 & 1517.406 & -2593.903 & -1208.430 \\
TARGET2 & 423.553 & -2524.940 & -1229.879 \\
TARGET3 & 1778.656 & -2635.034 & -872.662 \\
TARGET4 & 1551.505 & -2618.273 & -852.950 \\
\hline & 159 & & \\
\hline
\end{tabular}

Image processing effect based on Photogrammetry software.

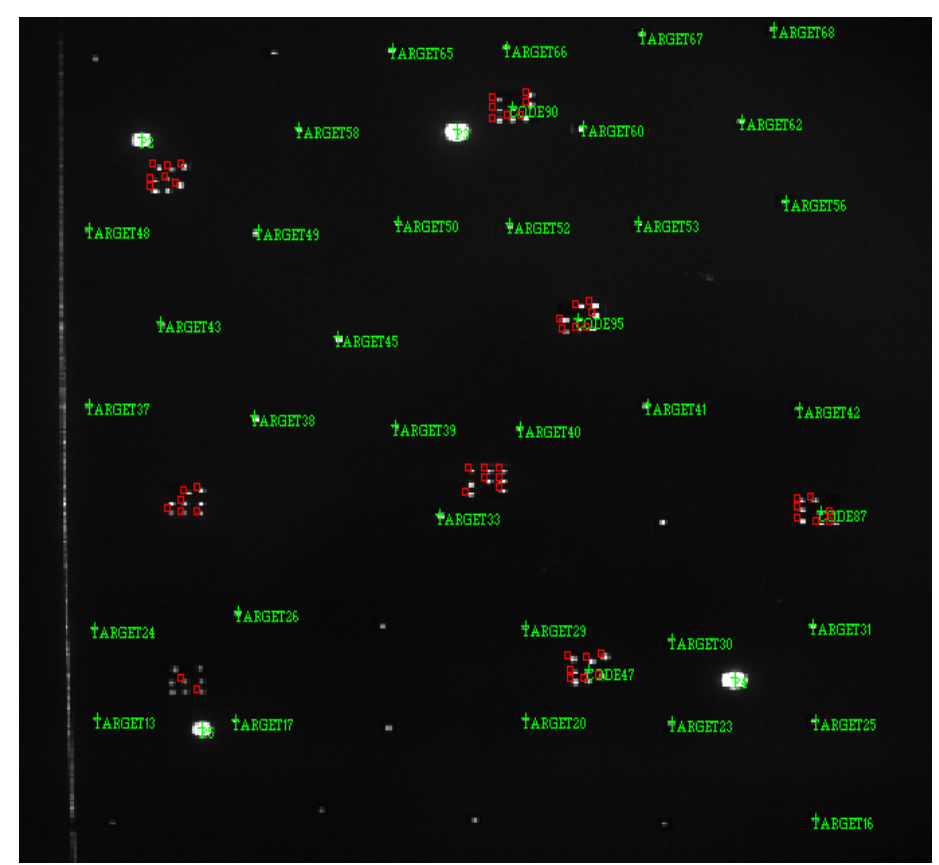

Fig. 6 Scanning recognition effect chart. 
Code points are the encoding point, while the P1-P4 as a common point, TARGET point common mark. It can be seen that the center of the center of the scanning and identifying center and the original mark point will have a little deviation, so that the internal parameters can be calibrated. Calibration software is used to calibrate the camera, and the internal and external parameters of the camera are obtained. The results of the internal parameters are as follows:

$\mathrm{C}=4.056029, \mathrm{XP}=-0.003482, \mathrm{YP}=0.000215, \mathrm{~K} 1=6.751373 \mathrm{e}-003, \mathrm{~K} 2=-3.431476 \mathrm{e}-004, \mathrm{~K} 3=$ $-1.306591 \mathrm{e}-006, \mathrm{P} 1=-5.4918 \mathrm{e}-004, \mathrm{P} 2=-3.823071 \mathrm{e}-004, \mathrm{AP} 1=-6.4192 \mathrm{e}-004$, AP2 $=3.502 \mathrm{e}-004$.

Table 2. The exterior orientation elements of the camera.

\begin{tabular}{ccccccc}
\hline 1 & 727.8543 & -787.3632 & -386.8259 & -1.55097225 & -0.05979132 & 3.13970889 \\
\hline 2 & 554.7205 & -864.3936 & -391.3458 & -1.55363668 & -0.24942658 & 3.11724855 \\
3 & 546.1780 & -803.3176 & -391.4050 & -1.55353351 & -0.26073157 & 3.11778629 \\
4 & 927.3552 & -822.2498 & -400.5881 & -1.58854160 & -0.13875101 & 3.11787258 \\
5 & 995.4379 & -840.8076 & -414.7602 & -1.54523549 & -0.07983420 & -3.08633398 \\
\hline
\end{tabular}

Fitting and transforming the points in the plane of the structure light, and get the parameters of the light plane in the camera coordinate system. As follows:

Table 3. Results of plane fitting settlement.

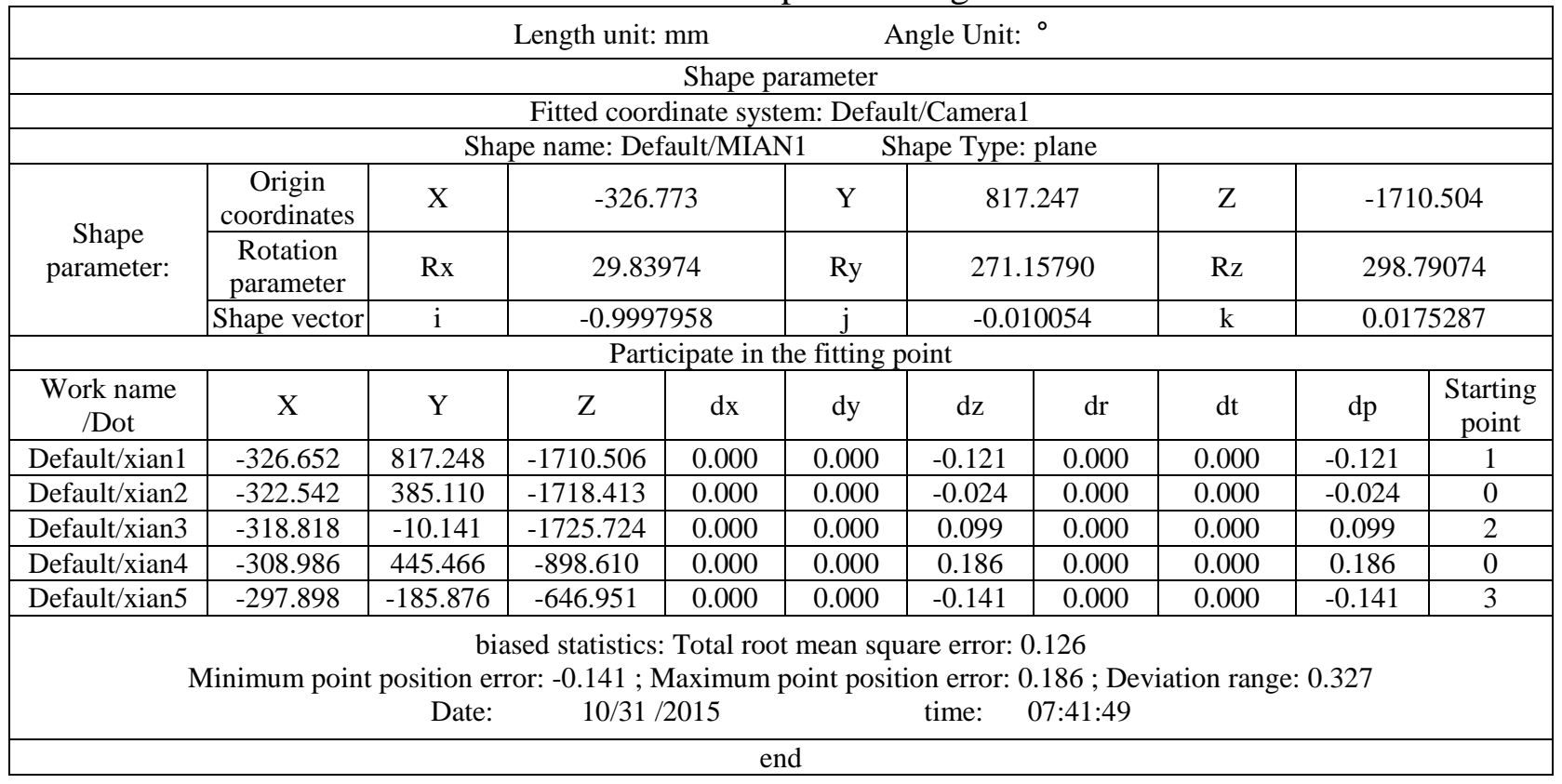

A total of five sets of data were measured, as follows:

Table 4. Light plane normal vector.

\begin{tabular}{cccc}
\hline & $\mathrm{I}$ & $\mathrm{j}$ & $\mathrm{k}$ \\
\hline 1 & -0.9998863 & -0.0110737 & 0.0102382 \\
2 & -0.9998909 & -0.0109902 & 0.0098715 \\
3 & -0.9998901 & -0.0111309 & 0.0097963 \\
4 & -0.9998877 & -0.011572 & 0.0095173 \\
5 & -0.9998859 & -0.0114039 & 0.0099078 \\
Mean & -0.99988818 & -0.01123414 & 0.00986622 \\
\hline
\end{tabular}

The average value is obtained, and the vector of the light plane method is obtained.

In the experiment, five groups were measured, five groups of normal vectors were obtained, for the difference between the two, the difference between the vector is $40.23625904,20.35478987$, $111.6720476,103.4417772,63.40573379$, unit is seconds. Mean value is 67.82212151 seconds, the mean error is 77.35 seconds.

With internal parameters for distortion correction of the camera, at the same time, the normal vector substitution, which combined into a structured light sensor system. The following calibration 
results are verified.

Taking a measurement of the length of the $200 \mathrm{~mm}$ cement box as the standard, taking pictures, get three sets of data, through the positioning endpoints and calculation, get the following results:

Table 5. Precision analysis result.

\begin{tabular}{cccc}
\hline Standard value & Measured value & Absolute error of measurement & Relative error \\
\hline 200.00000 & 200.865000 & 0.865000 & $0.433 \%$ \\
200.00000 & 200.426000 & 0.426000 & $0.213 \%$ \\
200.00000 & 199.865000 & 0.135000 & $0.068 \%$ \\
average & 200.385333 & 0.475333 & $0.238 \%$ \\
\hline
\end{tabular}

The results show that the average absolute error of sensing system $<0.48 \mathrm{~mm}$, and the average relative error is $0.24 \%$.

Factors that affect the measuring accuracy has the following points (1) The quality problem of the light plane emitted by the laser; (2) Center extraction of light stripe and image point of feature points location error; (3) The error caused by the surface roughness of the wall; (4) The camera internal parameters calibration error; (5) The vibration of the theodolite system will also affect the calibration accuracy. In addition, the analysis of the accuracy, light stripes and four points in a straight line misalignment will also affect the measurement results. By reducing the error, the measurement accuracy can be further improved.

\section{Conclusion}

In this paper, based on theodolite system, artificial materials of light reflecting, as well as the independent development of camera calibration software, using four point coplanar methods to get the plane equation of the light plane in the camera coordinate system. Experimental data show that, the sensing system can realize the relative measurement accuracy of about $0.24 \%$. The method is convenient and rapid to use the existing equipment and the software. By improving the experimental conditions, the calibration accuracy can be further improved.

\section{References}

[1] Q. G. Tian, B. Z. Ge, P. Du, Measurement of human figure size based on laser 3D scanning. Opt. Precis. Eng. 15(1) (2007) 84-88.

[2] Z. H. He, B. G. Wang, Model and imaging formula of the line structured light sensor. Opt. Precis. Eng. 9(3) (2001) 269-272.

[3] R. Dewar, Self-generated targets for spatial calibration of structured-light optical sectioning sensors with respect to an external coordinate system, Soc. Manuf. Eng. 1988.

[4] G. Y. Xu, L. F. Liu, J. C. Zeng, et al. A new method of calibration in 3D vision system based on structure light. Chinese J. Comput. 18(6) (1995) 450-456.

[5] D. Q. Huynh, R. A. Owens, P. E. Hartmann, Calibrating a Structured Light Stripe System: A Novel Approach, Int. J. Comput. Vis. 33(1) (1999) 73-86.

[6] F. M. Liu, F. J. Duan, S. H. Ye, New accurate calibration technique for the light-strip sensors, J. Tianjin University, 2(5) (1999) 547-550.

[7] F. J. Duan, F. M. Liu, S. H. Ye, A new accurate method for the calibration of line structured light sensor. Chinese J. Sci. Instrum. 21(1) (2000) 108-113.

[8] G. P. Huang, Study on the key technologies of digital close range industrial photogrammetry and applifications. College of precision instrument and opto Electronics Engineering Tianjin University, 2005. 